\title{
THE CHRISTOFFEL PROBLEM BY FUNDAMENTAL SOLUTION OF THE LAPLACE EQUATION
}

\author{
QI-RUI LI, DONGRUI WAN, AND XU-JIA WANG
}

\begin{abstract}
The Christoffel problem is equivalent to the existence of convex solutions to the Laplace equation on the unit sphere $\mathbb{S}^{n}$. Necessary and sufficient conditions have been found by Firey [11] and Berg [4, using the Green function of the Laplacian on the sphere. Expressing the Christoffel problem as the Laplace equation on the entire space $\mathbb{R}^{n+1}$, we observe that the second derivatives of the solution can be given by the fundamental solutions of the Laplace equations. Therefore we find new and simpler necessary and sufficient conditions for the solvability of the Christoffel problem. We also study the $L_{p}$ extension of the Christoffel problem and provide sufficient conditions for the problem, for the case $p \geq 2$.
\end{abstract}

\section{INTRODUCTION}

Given a positive function $f$ on the unit sphere $\mathbb{S}^{n}$, the Christoffel problem concerns the existence of a closed convex body $\Omega \subset \mathbb{R}^{n+1}$ such that the sum of the principal curvature radii of $\mathcal{M}=\partial \Omega$ at $p$ is equal to $f(x)$, where $x$ is the unit outward normal of $\mathcal{M}$ at $p$. This problem has been studied by many authors [9, 5, 17, 15, 21, 1, 19]. Firey [11] and Berg 44 finally obtained necessary and sufficient conditions for the solvability of the problem. We refer the readers to [11, 12, 13, for more details on early works on the Christoffel problem. In particular a nice sufficient condition was found in [13].

Let $u(x)=\sup \{x \cdot p \mid p \in \Omega\}$, where $x \in \mathbb{S}^{n}$, be the support function of $\Omega$. It is well known that the eigenvalues of the Hessian matrix $U=:\left\{\nabla^{2} u(x)+u(x) I\right\}$ are the principal radii of $\mathcal{M}$ at $p$, where $\nabla$ denotes the derivative with respect to an orthonormal frame on $\mathbb{S}^{n}$. Hence Christoffel's problem is equivalent to the existence of convex solutions to

$$
\Delta_{\mathbb{S}^{n}} u+n u=f \text { on } \mathbb{S}^{n},
$$

where $\Delta_{\mathbb{S}^{n}}$ is the Laplacian on $\mathbb{S}^{n}$. A necessary and sufficient condition for the existence of solutions to (1.1) is that $f$ is orthogonal to the kernel of the operator $\Delta+n I$ on $\mathbb{S}^{n}$,

2010 Mathematics Subject Classification. 35J99, 53A99. 
namely

$$
\int_{\mathbb{S}^{n}} x_{i} f(x) d x=0, \quad i=1, \ldots, n+1 .
$$

The key point to solve Christoffel's problem is to find conditions such that the solution is convex, namely the matrix $U \geq 0$. Firey [11] derived the Green function $G_{\mathbb{S}^{n}}(x, y)$ of the operator $\Delta_{\mathbb{S}^{n}}+n I$ on $\mathbb{S}^{n}$. Hence the solution to (1.1) is given by $u(x)=\int_{\mathbb{S}^{n}} G_{\mathbb{S}^{n}}(x, y) f(y) d y$, and the matrix $U \geq 0$ is equivalent to

$$
\left\{\int_{\mathbb{S}^{n}} \partial_{x_{i}} G_{\mathbb{S}^{n}}(x, y) \partial_{y_{j}} f(y) d y\right\} \geq 0 .
$$

Unfortunately the Green function on $\mathbb{S}^{n}$ is not explicitly given, and the condition (1.3) is not easy to verify. We remark that $U \equiv 0$ if and only if $u$ is linear, i.e., $\Omega$ is a point. But we assume that $f>0$, so this case does not occur.

Berg [4] studied Christoffel's problem after Firey. He deduced a recursion relation on the dimension for the expression of solutions. In [12 the authors re-created Berg's recursion relation by a different method. But again the recursion formulas in [11, 12] are rather complicated, and not easy to verify. We will state the conditions of Firey [11] and Berg [4] in Remark 3.1 for reader's convenience.

In this paper we observe that the second derivatives of the solution can be expressed by the fundamental solution of the Laplacian operator on $\mathbb{R}^{n+1}$, instead of the Green function on $\mathbb{S}^{n}$. Therefore we found much simpler conditions for the convexity of solutions. More precisely, we extend $u$ to $\mathbb{R}^{n+1}$ such that it is homogeneous of degree 1 , and extend $f$ to $\mathbb{R}^{n+1}$ such that it is homogeneous of degree -1 . Then equation (1.1) is equivalent to

$$
\Delta u=f \text { on } \mathbb{R}^{n+1} .
$$

Note that $u$ and $f$ have a singular point at 0 , so that $u$ is a weak solution in $W_{l o c}^{2, p}\left(\mathbb{R}^{n+1}\right)$ for $p \in(1, n)$. It is amazing to see that Christoffel's problem is equivalent to the existence of convex solutions to such a simple equation (1.4). Note that if $u$ is a convex solution to (1.4), then $\mathcal{M}$ can be recovered from $u$ by $\mathcal{M}=\left\{D u(x) \mid x \in \mathbb{S}^{n}\right\}$.

If $n=1, \mathcal{M}$ is a curve in $\mathbb{R}^{2}$. In this case, $U(x)=f(x)$ and the solution is convex if and only if $f(x) \geq 0$ but $f \not \equiv 0$. In the following we focus on the case $n \geq 2$. Denote

$$
w_{R}(x)=\int_{B_{R}(0)} F(x, y) f(y) d y,
$$

where $F(x, y)$ is the fundamental solution of the Laplace equation in $\mathbb{R}^{n+1}$,

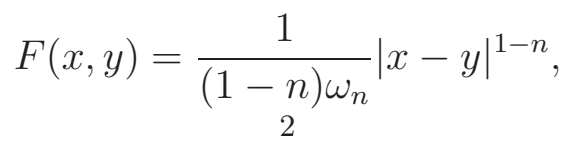


and $\omega_{n}=\left|\mathbb{S}^{n}\right|$. For any fixed $x$, when $R$ is sufficiently large, we have $F(x, y) f(y)=$ $O\left(|y|^{-n}\right)$. Hence $w_{R}=O(R)$ in $B_{R}(0)$. Noting that $u$ is homogeneous of degree 1 , the harmonic function $h_{R}=: u-w_{R}=O(R)$ in $B_{R}(0)$. Hence we have $D h_{R}=O(1)$ and $D^{2} h_{R}=O\left(R^{-1}\right)$ in $B_{R}(0)$. Therefore if the limits $w_{i j}(x)=: \lim _{R \rightarrow \infty} \frac{\partial^{2} w_{R}}{\partial x_{i} \partial x_{j}}(x)$ exist, we have

$$
u_{x_{i} x_{j}}(x)=w_{i j}(x) \quad \forall x \in \mathbb{R}^{n+1} .
$$

Note that on the right hand side, $i, j$ are understood as subscripts, not derivatives.

We compute the first derivative of $w_{R}$,

$$
\begin{aligned}
D_{i} w_{R}(x) & =\int_{B_{R}(0)} f(y) F_{x_{i}}(x, y) d y=-\int_{B_{R}(0)} f(y) F_{y_{i}}(x, y) d y \\
& =-\int_{\partial B_{R}(0)} f(y) F(x, y) \gamma_{i}+\int_{B_{R}(0)} f_{y_{i}} F(x, y) d y,
\end{aligned}
$$

and the second derivatives

$$
D_{i j} w_{R}(x)=-\int_{\partial B_{R}(0)} f(y) F_{x_{j}}(x, y) \gamma_{i}+\int_{B_{R}(0)} f_{y_{i}} F_{x_{j}}(x, y) d y,
$$

where $\gamma$ is the unit normal to $\partial B_{R}(0)$. The first integral in (1.9)

$$
\int_{\partial B_{R}} f(y) F_{x_{j}}(x, y) \gamma_{i}=\frac{1}{\omega_{n}} \int_{\partial B_{R}} \frac{f(y)\left(x_{j}-y_{j}\right) \gamma_{i}}{|x-y|^{n+1}}=O\left(R^{-1}\right) \rightarrow 0 .
$$

Hence

$$
\begin{aligned}
\lim _{R \rightarrow \infty} D_{i j} w_{R}(x) & =\int_{\mathbb{R}^{n+1}} f_{y_{i}} F_{x_{j}}(x, y) d y \\
& =\frac{1}{\omega_{n}} \int_{\mathbb{R}^{n+1}} \frac{\left(x_{j}-y_{j}\right) f_{y_{i}}}{|x-y|^{n+1}}=: w_{i j}(x) .
\end{aligned}
$$

For any point $x \in \mathbb{S}^{n}$, in order that $u$ is convex at $x$, by the homogeneity of $u$, it suffices to verify that for any unit vector $\xi=\left(\xi_{1}, \cdots, \xi_{n+1}\right)$ satisfying $\xi \perp x$, there holds $\sum_{i, j=1}^{n+1} w_{i j} \xi_{i} \xi_{j} \geq 0$. Therefore we obtain the following criterion for the convexity of solutions to (1.4).

Theorem 1.1. Suppose $n \geq 2$ and $f$ is a positive and Lipschitz continuous function. The solution $u$ is convex if and only if $\forall x \in \mathbb{S}^{n}$ and $\forall$ unit vector $\xi \perp x$, there holds

$$
\int_{\mathbb{R}^{n+1}} \frac{-\langle\xi, y\rangle f_{\xi}}{|x-y|^{n+1}} d y \geq 0 .
$$

Our condition (1.11) looks much simpler than (1.3), because the Green function on the sphere is very complicated. But we should point out that our condition is equivalent to Firey's, and also Berg's conditions, as they are all necessary and sufficient conditions 
for the Christoffel problem, see Remark 3.2. In Theorem 3.13.4 below, we will give sufficient conditions for Hölder continuous $f$. Note when $n=1, \mathcal{M}$ is a closed curve and is automatically convex when $f>0$.

We point out that condition (1.11) is only used for the convexity of solution. For the existence of solutions to (1.1), one also needs to assume the condition (1.2) as in [11]. Combining the existence of solutions in [11] and the convexity of solutions (Theorem 1.1), we have

Theorem 1.2. Suppose $n \geq 2$ and $f$ is a positive Lipschitz continuous function. The Christoffel problem has a solution if and only if $f$ satisfies (1.2) and (1.11). Moreover, the solution is unique up to translation.

This paper is divided into two parts. In part I, we will discuss in more details on the condition (1.11). For example, by integration by parts, we see that the solution is convex if $f$ is Hölder continuous and its Hölder norm is smaller than a certain constant. The constant is computed in (3.4). We will also deduce other sufficient conditions on $f$ such that the solution is convex. These conditions are contained in Theorems 3.1]3.4. In Section 2 we also give a proof for the existence of entire solutions to equation (1.4), for any locally integrable function $f$.

In part II, we consider an extension of the Christoffel problem, called the $L_{p}$-Christoffel problem. The associated equation is

$$
\Delta_{S^{n}} u+n u=f(x) u^{p-1} \text { on } \mathbb{S}^{n} .
$$

This equation was first introduced in [16] and later studied in [14]. In these two papers, the authors extended the sufficient condition for the Christoffel-Minkowski problem in [13] to equation (1.12), for $p>1$. In this paper we give some sufficient conditions for the convexity of solutions to (1.12) in Theorem 4.1.

\section{Existence of Entire solutions to the LAplace EQuation}

In this section, we show that equation (1.4) has a solution for any locally integrable $f$. This is a known result but it is hard to find a proof in literature. So we present a proof here which should be of interest to the readers.

Theorem 2.1. Suppose $f(x) \in L_{l o c}^{p}\left(\mathbb{R}^{n}\right)$ for some $p \geq 1$. Then there exists a solution $u$ to the equation

$$
\Delta u=f(x) \text { on } \mathbb{R}^{n}
$$


Proof. At first we consider the 2-dimensional case and suppose $f(x)$ is a locally bounded function. Let

$$
\begin{aligned}
G(x, y) & =: \frac{1}{2 \pi}(\log |x-y|-\log |y|) \\
& =\frac{1}{4 \pi} \log \left(1+\frac{|x|^{2}-2 x \cdot y}{|y|^{2}}\right) \\
& =-\frac{1}{4 \pi} \sum_{j=1}^{\infty} \frac{1}{j}\left(\frac{2 x \cdot y-|x|^{2}}{|y|^{2}}\right)^{j} \\
& =\sum_{|\alpha| \geq 1} \phi_{\alpha}(y) x^{\alpha},
\end{aligned}
$$

where $\alpha=\left(\alpha_{1}, \ldots, \alpha_{n}\right)$ with $\alpha_{i} \geq 0, x^{\alpha}=\prod_{i=1}^{n} x_{i}^{\alpha_{i}}$, and $\phi_{\alpha}(y)$ satisfies

$$
\phi_{\alpha}(r y)=\frac{1}{r^{|\alpha|}} \phi_{\alpha}(y) \text {. }
$$

Since $\Delta_{x} G(x, y)=0$, we have

$$
\Delta_{x}\left(\sum_{|\alpha|=k} \phi_{\alpha}(y) x^{\alpha}\right)=0 \quad \forall k \geq 1 .
$$

Moreover,

$$
\left|\phi_{\alpha}(y)\right| \leq M^{|\alpha|} /|y|^{|\alpha|}
$$

for some $M>0$ independent of $|\alpha|$.

Let

$$
\psi_{k}(x, y)=\sum_{|\alpha| \leq k} \phi_{\alpha}(y) x^{\alpha}, \quad k \geq 1
$$

We can select a monotone increasing sequence $R_{k} \rightarrow \infty$ as $k \rightarrow \infty$ so that

$$
\sum_{|\alpha|=k}^{\infty}\left|\phi_{\alpha}(y)\right||x|^{|\alpha|} \leq \frac{1}{1+|y|^{n+2}} \frac{1}{\sup _{|x| \leq R_{k}}(1+|f(x)|)} \quad \forall 1<|y| \leq R_{k} \text { and }|x| \leq|y| / 4 M \text {. }
$$

This is possible since the left hand side

$$
\leq \sum_{|\alpha|=k}^{\infty} \frac{M^{|\alpha|}|x|^{|\alpha|}}{|y|^{|\alpha|}} \leq \sum_{|\alpha|=k}^{\infty} \frac{1}{4^{|\alpha|}} \leq \sum_{j=k}^{\infty} 2^{-j}
$$

provided $|y| \geq 4 M|x|$.

Let $\Omega_{k}=B_{R_{k}}(0) \backslash B_{R_{k-1}}(0)$. Let

$$
u_{k}(x)=\sum_{j=1}^{k} \int_{\Omega_{j}} f(y)\left[G(x, y)-\psi_{j}(x, y)\right] d y .
$$


Then

$$
\Delta u_{k}(x)=\left\{\begin{array}{lr}
f(x), & x \in B_{R_{k}}(0), \\
0, & |x|>R_{k} .
\end{array}\right.
$$

For $|x| \leq R_{k} / 4 M$, we estimate $u_{k}(x)$ as follows.

$$
\begin{aligned}
\left|u_{k}(x)\right| & \leq \sum_{j=1}^{j_{0}}\left|\int_{\Omega_{j}} f(y)\left[G(x, y)-\psi_{j}(x, y)\right] d y\right| \\
& +\sum_{j=j_{0}+1}^{k}\left|\int_{\Omega_{j}} f(y)\left[G(x, y)-\psi_{j}(x, y)\right] d y\right|
\end{aligned}
$$

where $j_{0}$ is the smallest integer so that $R_{j_{0}} \geq 4 M|x|\left(j_{0}\right.$ depends on $|x|$ and $f$, but is independent of $k$ ). The first term

$$
\begin{aligned}
& \leq \sum_{j=1}^{j_{0}}\left|\int_{\Omega_{j}} f(y)\left[G(x, y)-\psi_{j}(x, y)\right] d y\right| \\
& \leq C_{f, x} \int_{B_{R_{j_{0}}}(0)}|G(x, y)|+\sum_{j=1}^{j_{0}}\left|\psi_{j}(x, y)\right| d y \\
& \leq \tilde{C}_{f, x}
\end{aligned}
$$

The second term

$$
\begin{aligned}
& \leq \sum_{j=j_{0}+1}^{k}\left|\int_{\Omega_{j}} f(y) \frac{1}{1+|y|^{n+2}} \frac{1}{\sup _{|x| \leq R_{j}}(1+|f(x)|)} d y\right| \\
& \leq C \int_{B_{R_{k}}(0) \backslash B_{R_{j_{0}}}(0)} \frac{1}{1+|y|^{n+2}} d y \\
& \leq C .
\end{aligned}
$$

Hence for any given $|x| \leq R_{k} / 4 M$,

$$
u_{k}(x) \leq C
$$

with $C$ depending only on $x$ and $f$, but is independent of $k$. Passing to a subsequence we see that $u_{k}(x) \rightarrow u(x)$ as $k \rightarrow \infty$ and $u$ is a solution of (2.1). Moreover, $u(x)$ can be represented by

$$
u(x)=\sum_{j=1}^{\infty} \int_{\Omega_{j}} f(y)\left[G(x, y)-\psi_{j}(x, y)\right] d y .
$$

For general $f(x) \in L_{l o c}^{p}\left(\mathbb{R}^{n}\right)$, we may decompose $f(x)=g(x)+h(x)$ so that $g(x)$ is a locally bounded function and $h(x) \in L^{p}\left(\mathbb{R}^{n}\right)$. From the above proof there exists a 
solution to $\Delta v(x)=g(x)$. The existence of $\Delta w(x)=h(x)$ is known, see, e.g. [2]. Hence $v(x)+w(x)$ is a solution of (2.1).

For higher dimensional case, let

$$
\begin{aligned}
G(x, y) & =\frac{-1}{(n-2) \omega_{n-1}}\left\{|x-y|^{2-n}-|y|^{2-n}\right\} \\
& =\frac{-1}{(n-2) \omega_{n-1}|y|^{n-2}}\left\{\frac{1}{\left(1+\frac{|x|^{2}-2 x \cdot y}{|y|^{2}}\right)^{\frac{n-2}{2}}}-1\right\} \\
& =\sum_{|\alpha| \geq 1} \phi_{\alpha}(y) x^{\alpha}
\end{aligned}
$$

where $\phi_{\alpha}(y)$ satisfies

$$
\phi_{\alpha}(r y)=r^{2-n-|\alpha|} \phi_{\alpha}(y)
$$

and

$$
\Delta_{x}\left(\sum_{|\alpha|=k} \phi_{\alpha}(y) x^{\alpha}\right)=0 \quad \forall k \geq 1
$$

Moreover,

$$
\left|\phi_{\alpha}(y)\right| \leq M^{|\alpha|} /|y|^{|\alpha|+n-2}
$$

for some $M>0$ independent of $|\alpha|$. Suppose $f(x) \in L_{l o c}^{\infty}\left(\mathbb{R}^{n}\right)$. Let

$$
\psi_{k}(x, y)=\sum_{|\alpha| \leq k} \phi_{\alpha}(y) x^{\alpha}, \quad k \geq 1,
$$

and select an increasing sequence $R_{k} \rightarrow \infty$ as $k \rightarrow \infty$ so that, for $k$ large,

$$
\sum_{|\alpha|=k}^{\infty}\left|\phi_{\alpha}(y)\right||x|^{|\alpha|} \leq \frac{1}{1+|y|^{n+2}} \frac{1}{\sup _{|x| \leq R_{k}}(1+|f(x)|)} \forall 1<|y| \leq R_{k} \text { and }|x| \leq|y| / 4 M \text {. }
$$

Let $\Omega_{k}=B_{R_{k}}(0) \backslash B_{R_{k-1}}(0)$ and let

$$
u_{k}(x)=\sum_{j=1}^{k} \int_{\Omega_{j}} f(y)\left[G(x, y)-\psi_{j}(x, y)\right] d y
$$

Similarly to the case $n=2$, we have $\left|u_{k}(x)\right| \leq C$ for $|x| \leq R_{k} / 4 M$, where $C$ is independent of $k$. Hence by selecting a subsequence we see that $u_{k}$ converges to a solution of (2.1).

We point out that equation (1.4) has a solution even if $f$ is not homogeneous of degree -1 . But in this case, the solution is not homogeneous of degree 1 . 


\section{Convex solutions to Christoffel's PRoblem}

In this section, we deduce more conditions on $f$ such that the solution to (1.4) is convex.

First by (1.9), we have

$$
\begin{aligned}
D_{i j} w_{R}(x)= & -\int_{\partial B_{R}(0)} f(y) F_{x_{j}}(x, y) \gamma_{i}+f(x) \int_{B_{R}(0)} F_{x_{j}}(x, y)\left(|y|^{-1}\right)_{y_{i}} d y \\
& +\int_{B_{R}(0)} F_{x_{j}}(x, y)\left(f(y)-|y|^{-1} f(x)\right)_{y_{i}} d y \\
= & -f(x) \int_{\partial B_{R}(0)}|y|^{-1} F_{x_{j}}(x, y) \gamma_{i}+f(x) \int_{B_{R}(0)} F_{x_{j}}(x, y)\left(|y|^{-1}\right)_{y_{i}} d y \\
& +\int_{B_{R}(0)} F_{x_{i} x_{j}}(x, y)\left(f(y)-|y|^{-1} f(x)\right) d y .
\end{aligned}
$$

The first integral in (3.1)

$$
\int_{\partial B_{R}(0)}|y|^{-1} F_{x_{j}}(x, y) \gamma_{i}=\frac{1}{\omega_{n} R} \int_{\partial B_{R}(0)} \frac{x_{j}-y_{j}}{|x-y|^{1+n}} \gamma_{i}=O\left(R^{-1}\right) .
$$

Noting that $u_{0}(x)=: \frac{1}{n}|x|$ solves (1.4) for $f=|x|^{-1}$, we can calculate the second integral in (3.1),

$$
\lim _{R \rightarrow \infty} \int_{B_{R}(0)} F_{x_{j}}(x, y)\left(|y|^{-1}\right)_{y_{i}} d y=\frac{1}{n}\left(\frac{\delta_{i j}}{|x|}-\frac{x_{i} x_{j}}{|x|^{3}}\right) .
$$

Sending $R \rightarrow \infty$, we conclude by (1.7)

$$
u_{i j}(x)=\frac{f(x)}{n}\left(\frac{\delta_{i j}}{|x|}-\frac{x_{i} x_{j}}{|x|^{3}}\right)+\int_{\mathbb{R}^{n+1}} F_{x_{i} x_{j}}(x, y)\left(f(y)-|y|^{-1} f(x)\right) d y .
$$

Note that the integral (3.2) is convergent provided $f \in C^{\alpha}\left(\mathbb{S}^{n}\right)$ for some $\alpha>0$. By the homogeneity, $u$ is convex if and only if $\sum_{i, j} u_{i j}(x) \xi_{i} \xi_{j} \geq 0$ for all unit vector $\xi$ satisfying $\xi \perp x$. Direct computation shows

$$
F_{x_{i} x_{j}}(x, y)=\frac{1}{\omega_{n}} \frac{|x-y|^{2} \delta_{i j}-(n+1)\left(x_{i}-y_{i}\right)\left(x_{j}-y_{j}\right)}{|x-y|^{n+3}} .
$$

Therefore we obtain the following criterion for the convexity of $u$.

Theorem 3.1. Let $n \geq 2$, and $f \in C^{\alpha}\left(\mathbb{S}^{n}\right)$ be a positive function. Extend $f$ to $\mathbb{R}^{n+1}$ such that it is homogeneous of degree -1. Then the solution $u$ to (1.1) is convex if and only if $\forall x, \xi \in \mathbb{S}^{n}$ with $\xi \perp x$,

$$
\frac{1}{\omega_{n}} \int_{\mathbb{R}^{n+1}} \frac{|x-y|^{2}-(n+1)\langle\xi, y\rangle^{2}}{|x-y|^{n+3}}(f(y)-f(x) /|y|) d y+\frac{1}{n} f(x) \geq 0 .
$$


From (3.3), we can give a quantitative condition on $f$ such that (1.1) admits a convex solution. For $\alpha>0$, denote

$$
\gamma_{n, \alpha}=\frac{\omega_{n}}{n(n+1)}\left[\int_{\mathbb{R}^{n+1}}\left|y-\mathbf{e}_{n+1}\right|^{-n-1}|y|^{-1} \operatorname{dist}_{\mathbb{S}^{n}}^{\alpha}\left(y /|y|, \mathbf{e}_{n+1}\right) d y\right]^{-1},
$$

where $\operatorname{dist}_{\mathbb{S}^{n}}(x, z)$ is the spherical distance between two points $x, z \in \mathbb{S}^{n}$, and $\mathbf{e}_{n+1}=$ $(0, \ldots, 0,1)$. Note that $\gamma_{n, \alpha}$ is unchanged if $\mathbf{e}_{n+1}$ is replaced by any other $x \in \mathbb{S}^{n}$.

Theorem 3.2. Let $n \geq 2$, and $f \in C^{\alpha}\left(\mathbb{S}^{n}\right)$ be a positive function, $\alpha \in(0,1)$. Then the solution to (1.1) is convex provided

$$
|f|_{C^{\alpha}\left(\mathbb{S}^{n}\right)} \leq \gamma_{n, \alpha} \min _{\mathbb{S}^{n}} f
$$

Proof. For $x, \xi \in \mathbb{S}^{n}$ with $\xi \perp x$ and $y \in \mathbb{R}^{n+1}$, we have

$$
\langle\xi, y\rangle^{2}=\langle\xi, y-x\rangle^{2} \leq|x-y|^{2},
$$

with equality if and only if $y-x=t \xi$ for some $t \in \mathbb{R}$. Therefore

$$
\begin{aligned}
& \text { LHS of }\left(\underline{3.3)}>-\frac{(n+1)|f|_{C^{\alpha}\left(\mathbb{S}^{n}\right)}}{\omega_{n}} \int_{\mathbb{R}^{n+1}}|x-y|^{-n-1}|y|^{-1} \operatorname{dist}_{\mathbb{S}^{n}}^{\alpha}(y /|y|, x) d y+\frac{1}{n} \min _{\mathbb{S}^{n}} f\right. \\
& \geq 0
\end{aligned}
$$

provided (3.5) holds.

By rewriting (1.11) and (3.3) in their equivalent form, we have the following theorem.

Theorem 3.3. Let $n \geq 2, f>0$ be a function on $\mathbb{S}^{n}$. Extend $f$ to $\mathbb{R}^{n+1}$ such that it is homogeneous of degree -1 .

(i) If $f \in C^{0,1}\left(\mathbb{S}^{n}\right)$, then the solution $u$ to (1.1) is convex if and only if

$$
\int_{\mathbb{S}^{n}} \omega(x, z)\langle\xi, z\rangle\langle D f(z), \xi\rangle d z \geq 0 \quad \forall x, \xi \in \mathbb{S}^{n} \text { satisfying } x \perp \xi,
$$

where

$$
\omega(x, z)=-\int_{0}^{\infty} \frac{r^{n-1}}{|x-r z|^{n+1}} d r .
$$

(ii) If $f \in C^{\alpha}\left(\mathbb{S}^{n}\right)$ for some $\alpha \in(0,1)$, then the solution $u$ is convex if and only if

$$
\int_{\mathbb{S}^{n}} \hat{\omega}(x, z, \xi) g(x, z) d \sigma_{\mathbb{S}^{n}}(z)+\frac{1}{n} f(x) \geq 0 \quad \forall x, \xi \in \mathbb{S}^{n} \text { satisfying } x \perp \xi,
$$

where

$$
\begin{aligned}
\hat{\omega}(x, \xi, z) & =\frac{1}{\omega_{n}} \int_{0}^{\infty} \frac{|x-r z|^{2}-(n+1)\langle\xi, r z\rangle^{2}}{|x-r z|^{n+3}} r^{n-1} d r, \\
g(x, z) & =f(z)-f(x) .
\end{aligned}
$$


Proof. We first show that (3.6) is necessary and sufficient for the convexity. Direct calculation shows that, by the homogeneity of $f$,

$$
\begin{aligned}
\text { LHS of (1.11) } & =-\int_{0}^{\infty} \int_{\mathbb{S}^{n}} \frac{r^{n-1}}{|x-r z|^{n+1}}\langle\xi, z\rangle\langle D f(z), \xi\rangle d r d z \\
& =\int_{\mathbb{S}^{n}} \omega(x, z)\langle\xi, z\rangle\langle D f(z), \xi\rangle d z .
\end{aligned}
$$

Note that

$$
\langle\xi, z\rangle=\langle\xi, z-x\rangle=O(|x-z|)
$$

We will verify that

$$
\omega(x, z)=O\left(|x-z|^{-n}\right) \quad \text { for } z \text { near } x .
$$

Hence the integral (3.8) is convergent, and by Theorem 1.1 we finish the proof of part (i). To verify (3.9). we assume by a rotation of coordinates that $x=\mathbf{e}_{n+1}$ and $z=$ $\left(\varepsilon \mathbf{e}_{1}+\mathbf{e}_{n+1}\right) / \sqrt{1+\varepsilon^{2}}$, where $\mathbf{e}_{k}$ is the unit vector on the $x_{k}$-axis. Then

$$
\begin{aligned}
\omega(x, z) & \simeq-\int_{0}^{2} \frac{r^{n-1}}{\left((1-r)^{2}+\varepsilon^{2} r^{2}\right)^{\frac{n+1}{2}}} d r \simeq-\int_{-1}^{1} \frac{(1+t)^{n-1}}{\left(t^{2}+\varepsilon^{2}\right)^{\frac{n+1}{2}}} d t \\
& \simeq-\varepsilon^{-n} \int_{-1 / \varepsilon}^{1 / \varepsilon} \frac{1}{\left(\rho^{2}+1\right)^{\frac{n+1}{2}}} d \rho \\
& =O\left(\varepsilon^{-n}\right) .
\end{aligned}
$$

Hence (3.9) follows.

We next prove part (ii). By computation,

$$
\begin{aligned}
\text { LHS of (3.3) } & =\frac{1}{\omega_{n}} \int_{\mathbb{S}^{n}} \int_{0}^{\infty} \frac{|x-r z|^{2}-(n+1)\langle\xi, r z\rangle^{2}}{|x-r z|^{n+3}} r^{n-1}(f(z)-f(x)) d r d z+\frac{1}{n} f(x) \\
& =\int_{\mathbb{S}^{n}} \hat{\omega}(x, \xi, z) g(x, z) d z+\frac{1}{n} f(x),
\end{aligned}
$$

where $g(x, z)=O\left(|x-z|^{\alpha}\right)$ as $f \in C^{\alpha}\left(\mathbb{S}^{n}\right)$. We will verify that

$$
\hat{\omega}(x, \xi, z)=O\left(|x-z|^{-n}\right) \text { for } z \text { near } x \text {. }
$$

Assume (3.11) for a moment. Then the integral in (3.10) is convergent and by Theorem 3.1, one sees that (3.7) is a necessary and sufficient condition for the convexity. To verify (3.11), we assume that $x=\mathbf{e}_{n+1}$ and $\xi=\mathbf{e}_{1}$. Fix a small $\varepsilon>0$ and consider 
$z_{1, \varepsilon}=\left(\varepsilon \mathbf{e}_{1}+\mathbf{e}_{n+1}\right) / \sqrt{1+\varepsilon^{2}}$. Then

$$
\begin{aligned}
\hat{\omega}\left(x, \xi, z_{1, \varepsilon}\right) & \simeq \int_{0}^{\infty} \frac{r^{n-1}}{\left((1-r)^{2}+\varepsilon^{2}\right)^{\frac{n+1}{2}}} d r-\varepsilon^{2} \int_{0}^{\infty} \frac{r^{n+1}}{\left((1-r)^{2}+\varepsilon^{2}\right)^{\frac{n+3}{2}}} d r \\
& \simeq \int_{-1}^{1} \frac{(t+1)^{n-1}}{\left(t^{2}+\varepsilon^{2}\right)^{\frac{n+1}{2}}} d t-\varepsilon^{2} \int_{-1}^{1} \frac{(t+1)^{n+1}}{\left(t^{2}+\varepsilon^{2}\right)^{\frac{n+3}{2}}} d t \\
& \simeq \varepsilon^{-n} \int_{-1 / \varepsilon}^{1 / \varepsilon} \frac{1}{\left(\rho^{2}+1\right)^{\frac{n+1}{2}}} d \rho-\varepsilon^{-n} \int_{-1 / \varepsilon}^{1 / \varepsilon} \frac{1}{\left(\rho^{2}+1\right)^{\frac{n+3}{2}}} d \rho \\
& \simeq \varepsilon^{-n} .
\end{aligned}
$$

If we consider $z_{2, \varepsilon}=\left(\varepsilon \mathbf{e}_{2}+\mathbf{e}_{n+1}\right) / \sqrt{1+\varepsilon^{2}}$, then similarly,

$$
\omega\left(x, z_{2, \varepsilon}, \xi\right) \simeq \int_{0}^{\infty} \frac{r^{n-1}}{\left((1-r)^{2}+\varepsilon^{2}\right)^{\frac{n+1}{2}}} d r \simeq \varepsilon^{-n} .
$$

Apparently (3.11) follows from (3.12) and (3.13).

Next we give another sufficient condition for the convexity of solutions to Christoffel's problem.

Theorem 3.4. Let $n \geq 2, f \in C^{0,1}\left(\mathbb{S}^{n}\right)$ be a positive function. Extend $f$ to $\mathbb{R}^{n+1}$ such that it is homogeneous of degree -1. Then the solution to (1.1) is convex, provided

$$
\partial_{\xi} f(x+t \xi)-\partial_{\xi} f(x-t \xi) \leq 0, \quad \forall t>0, \xi \in \mathbb{S}^{n}, x \in \mathbb{R}^{n+1} \text { with }\langle\xi, x\rangle=0 .
$$

Proof. By Theorem 1.1, it suffices to show that

$$
J(z, \xi)<0, \quad \forall z, \xi \in \mathbb{S}^{n} \text { with } \xi \perp z,
$$

where

$$
J(z, \xi)=\int_{\mathbb{R}^{n+1}} \frac{\langle\xi, y\rangle f_{\xi}(y)}{|z-y|^{n+1}} d y
$$

For any given such $z$ and $\xi$, we assume by a rotation of coordinates that $\xi=\mathbf{e}_{1}$ and $z=\mathbf{e}_{n+1}$. Let $u$ be the solution to (1.4). Let $\hat{u}(y)=u\left(-y_{1}, y^{\prime}\right)$ and $\hat{f}(y)=f\left(-y_{1}, y^{\prime}\right)$, where $y^{\prime}=\left(y_{2}, \cdots, y_{n+1}\right)$. Then $\hat{u}$ is the solution corresponding to $\hat{f}$, and $u_{11}\left(\mathbf{e}_{n+1}\right)=$ $\hat{u}_{11}\left(\mathbf{e}_{n+1}\right)$. Hence

$$
u_{11}\left(\mathbf{e}_{n+1}\right)=\frac{1}{2}[u+\hat{u}]_{11}\left(\mathbf{e}_{n+1}\right) .
$$

Consequently,

$$
J(z, \xi)=\frac{1}{2} \int_{\mathbb{R}^{n+1}} \frac{y_{1}\left(f_{y_{1}}(y)+\hat{f}_{y_{1}}(y)\right)}{\left|\mathbf{e}_{n+1}-y\right|^{n+1}} d y .
$$


From (3.14) it follows that

$$
f_{y_{1}}(y)+\hat{f}_{y_{1}}(y)=f_{y_{1}}\left(y_{1}, y^{\prime}\right)-f_{y_{1}}\left(-y_{1}, y^{\prime}\right) \leq 0, \text { for } y_{1}>0 .
$$

Replacing $y_{1}$ by $-y_{1}$, we infer

$$
f_{y_{1}}(y)+\hat{f}_{y_{1}}(y)=-\left(f_{y_{1}}\left(-y_{1}, y^{\prime}\right)-f_{y_{1}}\left(y_{1}, y^{\prime}\right)\right) \geq 0, \text { for } y_{1}<0 .
$$

Combining the above two inequalities, we get

$$
y_{1}\left(f_{y_{1}}(y)+\hat{f}_{y_{1}}(y)\right) \leq 0, \quad \forall y \neq 0 .
$$

This together with (3.16) shows that $J(\xi, z) \leq 0$.

Suppose $J(z, \xi)=0$. Then (3.16) and (3.17) implies

$$
\partial_{y_{1}}\left(f\left(y_{1}, y^{\prime}\right)+f\left(-y_{1}, y^{\prime}\right)\right)=f_{y_{1}}(y)+\hat{f}_{y_{1}}(y)=0 \text { for almost all } y \in \mathbb{R}^{n+1} \text {. }
$$

As a consequence, there is a function $h$ defined in $\mathbb{R}^{n}$ such that, for almost all $y^{\prime} \in \mathbb{R}^{n}$,

$$
f\left(y_{1}, y^{\prime}\right)+f\left(-y_{1}, y^{\prime}\right)=h\left(y^{\prime}\right)
$$

Since $f$ is homogeneous of degree negative one, we conclude that, by sending $y_{1} \rightarrow \infty$, $h\left(y^{\prime}\right)=0$. Therefore, for almost all $y^{\prime} \in \mathbb{R}^{n}$,

$$
f\left(y_{1}, y^{\prime}\right)+f\left(-y_{1}, y^{\prime}\right)=0 .
$$

This contradicts our assumption $f>0$. We complete the proof.

We finish this section by a few remarks.

Remark 3.1. Here we state the conditions of Firey [11] and Berg [4], in comparison with our condition (1.11).

In [11], Firey reduced condition (1.3) to

$$
\int_{S^{n}}\left\langle x, y^{\prime}\right\rangle \Theta(x, y)\left\langle D f(x), y^{\prime}\right\rangle d x \leq 0 \quad \forall y, y^{\prime} \in \mathbb{S}^{n}, y \perp y^{\prime},
$$

where

$$
\Theta(x, y)=\left(1-\langle x, y\rangle^{2}\right)^{-n / 2} \int_{\pi}^{\arccos (\langle x, y\rangle)} \sin ^{n-1} t d t .
$$

Berg proved that the solution is convex if and only if the function

$$
\int_{S^{n}} g_{n}(\langle x, y\rangle) f(x) d x
$$

is convex, where

$$
\begin{aligned}
g_{2}(t) & =\frac{1}{\pi}\left(\pi-\cos ^{-1} t\right)\left(1-t^{2}\right)^{1 / 2}-\frac{t}{2 \pi} \\
g_{3}(t) & =1+t \log (1-t)+\left(\frac{4}{3}-\log 2\right) t
\end{aligned}
$$


and for $n \geq 2$,

$$
g_{n+2}(t)=\frac{n+1}{(n-1)^{2}} t g_{n}^{\prime}(t)+\frac{n+1}{n-1} g_{n}(t)+\frac{t}{\sqrt{\pi}} \frac{(n+1) \Gamma((n+2) / 2)}{(n+2) \Gamma((n+1) / 2)} .
$$

Our condition (1.11) is equivalent to (3.18) or (3.19), as all these conditions are derived from the fundamental solution. The conditions (3.18) and (3.19) are derived from the fundamental solution in $\mathbb{S}^{n}$ but ours is from that on $\mathbb{R}^{n}$. So our condition (1.11) looks simpler. Moreover, from our condition (1.11), we can derive a number of simpler sufficient conditions for the convexity of solutions. It is not so easy to find sufficient conditions from (3.18) and (3.19).

Remark 3.2. Firey's condition (3.18) is equivalent to our condition (1.11). Here we show how (3.18) can be derived from (1.11). Let $s=\langle x, z\rangle$ and $\theta=\arccos s$. It is not hard to see that

$$
\omega(x, z)=-\int_{0}^{\infty} \frac{r^{n-1}}{\left(r^{2}-2 s r+1\right)^{\frac{n+1}{2}}} d r
$$

where $\omega(x, z)$ is given in Theorem 3.3 . Denote $\rho=(r-s) / \sqrt{1-s^{2}}$. Then we have

$$
\omega(x, z)=-\left(1-s^{2}\right)^{-\frac{n}{2}} \int_{-\frac{s}{\sqrt{1-s^{2}}}}^{\infty} \frac{\left(\sqrt{1-s^{2}} \rho+s\right)^{n-1}}{\left(\rho^{2}+1\right)^{\frac{n+1}{2}}} d \rho .
$$

Taking $\rho=-\cot \varphi$ and using $s=\cos \theta$, we further deduce

$$
\begin{aligned}
\omega(x, z) & =-\left(1-s^{2}\right)^{-\frac{n}{2}} \int_{\theta}^{\pi} \sin ^{n-1}(\varphi-\theta) d \varphi \\
& =\left(1-\langle x, z\rangle^{2}\right)^{-\frac{n}{2}} \int_{\pi}^{\arccos \langle x, z\rangle} \sin ^{n-1} t d t .
\end{aligned}
$$

Hence our condition (3.6) is equivalent to Firey's condition (3.18).

Remark 3.3. Pogorelov [19] established the convexity of solutions to (1.1) when $n=2$ under the condition

$$
f(x)-f_{s s}(x)>0 \text { on } \mathbb{S}^{2},
$$

where the sub-script $s$ means differentiation with respect to arc length of great circle on $\mathbb{S}^{2}$. In [13], Guan and Ma studied the Christoffel-Minkowski problem, which is to find convex bodies with prescribed area measure of order $k$. When $k=1$, it is the Christoffel problem discussed in this paper. By proving a constant rank theorem they found the following sufficient condition for the convexity of solutions to (1.1),

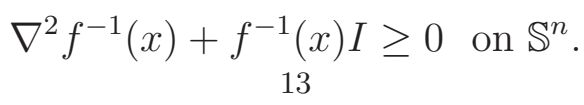


It is easy to see that if $f$ satisfies (3.20), then it satisfies (3.21), for two dimensions.

Our conditions in Theorem 1.1 and in Theorems 3.1-3.4 do not involve the second derivatives of $f$, and so are different from (3.21).

\section{The $L_{p}$ Christoffel Problem}

In this section we study an extension of the Christoffel problem, called the $L_{p}$ Christoffel problem. The problem was introduced by $\mathrm{Hu}$, Ma and Shen [16], and studied later by Guan and Xia [14. It can be formulated as finding convex solutions to the equation (1.12).

In [16, 14], a constant rank theorem was established for convex solutions to (1.12), provided that $f \in C^{1,1}\left(\mathbb{S}^{n}\right), f>0$, and

$$
\nabla^{2} f^{-\frac{1}{p}}+f^{-\frac{1}{p}} I \geq 0 \text { on } \mathbb{S}^{n} .
$$

As a result, the existence of convex solutions to (1.12) was obtained in [16] for the case $p \geq 2$; and an even convex solution to (1.12) was obtained in [14] if $f$ is also even and $1<p<2$. The papers [16] and [14] also obtained similar results for the $L_{p}$ version of the Christoffel-Minkowski problem.

Equation (1.12) is a semi-linear elliptic equation. Semi-linear elliptic equations have been extensively studied in the last four decades. An example is the prescribed scalar curvature eqjuation on $\mathbb{S}^{n}$,

$$
-\Delta_{\mathbb{S} n} u+\frac{n(n-2)}{4} u=\frac{n-2}{4(n-1)} u^{\frac{n+2}{n-2}} f \text { on } \mathbb{S}^{n}, n>2 .
$$

Equation (4.2) has been studied by numerous authors in the last a few decades, see e.g. [3, 6, 17, 8, 10, 18. But the $L_{p}$-Christoffel problem, though the equation is so simple, received much less attention. In this paper we will prove the following result.

Theorem 4.1. Let $n \geq 2$, and $f \in C^{0,1}\left(\mathbb{S}^{n}\right)$ be a positive function. Given $p \geq 2$, if

$$
\left(1+\frac{n(p-1)}{n-1} \frac{\max _{\mathbb{S}^{n}} f}{\min _{\mathbb{S}^{n}} f}\right)\left[\exp \left(\frac{n \pi}{n-1} \frac{\max _{\mathbb{S}^{n}}|\nabla f|}{\min _{\mathbb{S}^{n}} f}\right)\right]^{p-1} \max _{\mathbb{S}^{n}}|\nabla f| \leq \gamma_{n, 1} \min _{\mathbb{S}^{n}} f,
$$

then

(i) when $p>2$, there is a unique positive convex solution $u$ to (1.12).

(ii) when $p=2$, there is a unique $\lambda>0$ such that (1.12) with $f$ replaced by $\lambda f$ has a unique (up to dilations) positive convex solution $u$. 
When $p \geq 2$, the existence and uniqueness of positive solutions were proved in [16]. We only need to show that the solution is convex under the condition (4.3). Note that (4.3) is for the convexity, but not for the existence and uniqueness of solutions.

We first present a lemma on the gradient estimate for the solutions to (1.12), in the case $p \geq 2$.

Lemma 4.1. Let $p \geq 2, f \in C^{0,1}\left(\mathbb{S}^{n}\right)$ be a positive function, and $u$ be a positive $C^{2}$ solution to (1.12). Then

$$
\max _{\mathbb{S}^{n}} \frac{|\nabla u|}{u} \leq \frac{n}{n-1} \frac{\max _{\mathbb{S}^{n}}|\nabla f|}{\min _{\mathbb{S}^{n}} f} .
$$

Proof. Let $v=\log u$. Since $u$ is solution to (1.12), we have

$$
\Delta_{\mathbb{S}^{n}} v+|\nabla v|^{2}+n=f e^{(p-2) v} \text { on } \mathbb{S}^{n} .
$$

Let $Q=|\nabla v|^{2}$. Suppose that $Q$ attains its maximum at $x_{0}$. By a rotation of the coordiantes, we may assume that $|\nabla v|\left(x_{0}\right)=v_{1}\left(x_{0}\right)$. Hence

$$
0=\nabla_{1} Q\left(x_{0}\right)=2 v_{1} v_{11} .
$$

Differentiating (4.4) yields, at $x_{0}$,

$$
\sum_{i} v_{i i 1}=\left(\nabla_{1} f+(p-2) f v_{1}\right) e^{(p-2) v} \geq \nabla_{1} f u^{p-2} .
$$

Then, by using the Ricci identity,

$$
\begin{aligned}
0 \geq \Delta_{\mathbb{S}^{n}} Q\left(x_{0}\right) & =2 \sum_{i, j} v_{j i i} v_{j}+2 \sum_{i, j} v_{i j}^{2} \\
& =2 \sum_{i, j}\left(v_{i i j}+v_{j}-v_{i} \delta_{i j}\right) v_{j}+2 \sum_{i, j} v_{i j}^{2} \\
& \geq 2 v_{1} \sum_{i} v_{i i 1}+2(n-1) v_{1}^{2} \\
& \geq 2 v_{1} \nabla_{1} f u^{p-2}+2(n-1) v_{1}^{2},
\end{aligned}
$$

where (4.5) is used in the last inequality. We also have

$$
u^{p-2}\left(x_{0}\right) \leq\left(\max _{\mathbb{S}^{n}} u\right)^{p-2} \leq n / \min _{\mathbb{S}^{n}} f,
$$

where the second inequality above follows by applying the maximum principle to (1.12). Plugging (4.7) into (4.6), we get

$$
0 \geq-2 n v_{1} \frac{\max _{\mathbb{S}^{n}}|\nabla f|}{\min _{\mathbb{S}^{n}} f}+2(n-1) v_{1}^{2} .
$$

Since $v_{1}\left(x_{0}\right)=\max _{\mathbb{S}^{n}} \frac{|\nabla u|}{u}$, we complete the proof by (4.8). 
We next finish the proof of Theorem 4.1.

Proof. As aforementioned, the existence and uniqueness of positive solutions in Theorem 4.1 were obtained in [16]. We only need to prove the convexity of the solution $u$.

We extend $u$ in $\mathbb{R}^{n+1}$ such that it is homogeneous of degree 1 , and extend $f$ in $\mathbb{R}^{n+1}$ such that it is homogeneous of degree $-p$. Then $f u^{p-1}$, after extension, is a homogeneous function of degree -1 . For convenience we still use $u$ and $f$ to denote their extensions. Then $u$ satisfies equation (1.4) with RHS replaced by $f u^{p-1}$ when $p>2$, or $\lambda f u^{p-1}$ when $p=2$.

Let us first prove part (i) of Theorem 4.1, Replacing $f$ by $f u^{p-1}$ in (3.2), we obtain, for any $x, \xi \in \mathbb{S}^{n}$ with $x \perp \xi$,

$$
\begin{aligned}
\sum u_{i j}(x) \xi_{i} \xi_{j}= & \frac{1}{n} f u^{p-1}(x)+u^{p-1}(x) \int_{\mathbb{R}^{n+1}} F_{\xi \xi}(x, y)|y|^{-1}(f(y /|y|)-f(x)) d y \\
& +\int_{\mathbb{R}^{n+1}} F_{\xi \xi}(x, y)|y|^{-1}\left(u^{p-1}(y /|y|)-u^{p-1}(x)\right) f(y /|y|) d y \\
\geq & \frac{1}{n} m_{u}^{p-1} \min _{\mathbb{S}^{n}} f-M_{u}^{p-1} \max _{\mathbb{S}^{n}}|\nabla f| \int_{\mathbb{R}^{n+1}}\left|F_{\xi \xi}(x, y)\right||y|^{-1} \operatorname{dist}_{\mathbb{S}^{n}}(y /|y|, x) d y \\
& -(p-1) M_{u}^{p-2} \max _{\mathbb{S}^{n}}|\nabla u| \max _{\mathbb{S}^{n}} f \int_{\mathbb{R}^{n+1}}\left|F_{\xi \xi}(x, y)\right||y|^{-1} \operatorname{dist}_{\mathbb{S}^{n}}(y /|y|, x) d y .
\end{aligned}
$$

where $M_{u}=\max _{\mathbb{S}^{n}} u, m_{u}=\min _{\mathbb{S}^{n}} u$, and

$$
\begin{aligned}
F_{\xi \xi}(x, y) & =\sum \xi_{i} \xi_{j} F_{x_{i} x_{j}}(x, y) \\
& =\frac{|x-y|^{2}-(n+1)\langle\xi, y\rangle^{2}}{\omega_{n}|x-y|^{n+3}} .
\end{aligned}
$$

Since $\langle\xi, y\rangle^{2}=\langle\xi, y-x\rangle^{2} \leq|x-y|^{2}$, we have

$$
\begin{aligned}
\left|F_{\xi \xi}(x, y)\right| & \leq \frac{1}{\omega_{n}|x-y|^{n+3}} \max \left\{|x-y|^{2},(n+1)\langle\xi, y\rangle^{2}\right\} \\
& \leq \frac{(n+1)}{\omega_{n}|x-y|^{n+1}} .
\end{aligned}
$$

Plugging (4.10) into (4.9), and noting that strict inequality holds in (4.10) when $y \neq$ $x+t \xi, t \in \mathbb{R}$, we obtain

$\sum u_{i j}(x) \xi_{i} \xi_{j}>\frac{1}{n} m_{u}^{p-1} \min _{\mathbb{S}^{n}} f-\frac{1}{n \gamma_{n, 1}}\left(M_{u}^{p-1} \max _{\mathbb{S}^{n}}|\nabla f|+(p-1) M_{u}^{p-2} \max _{\mathbb{S}^{n}}|\nabla u| \max _{\mathbb{S}^{n}} f\right)$,

where $\gamma_{n, 1}$ is given by (3.4) (taking $\alpha=1$ ). In view of Lemma 4.1,

$$
\max _{\mathbb{S}^{n}}|\nabla u| \leq M_{u} \frac{n}{n-1} \frac{\max _{\mathbb{S}^{n}}|\nabla f|}{\min _{\mathbb{S}^{n}} f} .
$$


Therefore

$$
\sum u_{i j}(x) \xi_{i} \xi_{j}>\left[\gamma_{n, 1} \min _{\mathbb{S}^{n}} f-\left(\frac{n(p-1)}{n-1} \frac{\max _{\mathbb{S}^{n}} f}{\min _{\mathbb{S}^{n}} f}+1\right)\left(\frac{M_{u}}{m_{u}}\right)^{p-1} \max _{\mathbb{S}^{n}}|\nabla f|\right] \frac{m_{u}^{p-1}}{n \gamma_{n, 1}} .
$$

Note that, by using Lemma 4.1 again,

$$
\begin{aligned}
\frac{M_{u}}{m_{u}} & \leq \exp \left(\pi \max _{\mathbb{S}^{n}} \frac{|\nabla u|}{u}\right) \\
& \leq \exp \left(\frac{n \pi}{n-1} \frac{\max _{\mathbb{S}^{n}}|\nabla f|}{\min _{\mathbb{S}^{n}} f}\right) .
\end{aligned}
$$

This together with (4.11) shows that $\sum u_{i j}(x) \xi_{i} \xi_{j}>0$, provided (4.3) holds. Hence the solution is convex.

For part (ii) of Theorem 4.1, we know that after homogeneous extension $u$ solves (1.1) with RHS being $\lambda f u^{p-1}$. Hence, replacing $f$ by $\lambda f$ in the above argument, we see immediately that the solution is convex under the condition (4.3).

\section{REFERENCES}

[1] Aleksandrov, A. D.: Über die Frage nach der Existenz eines konvexen Körpers bei dem die Summe der Hauptkrümmungsradien eine gegebene positive Funcktion ist, welche die Bedingungen der Geschlossenheit genügt. Dokl. Akad. Nauk SSSR 14 (1937), 59-60.

[2] C. Amrouche, V. Girault, and J. Giroire, Weighted Sobolev spaces for Laplace's equations in $\mathbb{R}^{n}$, J. Math. Pures Appl. 73(1994), 579-606.

[3] Bahri, A.; Coron, J. M.: The scalar-curvature problem on three-dimensional sphere. J. Funct. Anal. 95 (1991), 106-172.

[4] Berg, C.: Corps convexes et potentiels sphériques. Danske Vid. Selskab, Mat.-fys. Medd. 37 (1969), $1-64$.

[5] Busemann, H., Convex surfaces, Interscience Tracts in Pure and Applied Mathematics, no. 6, Interscience Publishers, Inc., New York, 1958.

[6] Chang, S. Y. A.; Yang, P. C.: Prescribing Gaussian curvature on $\mathbb{S}^{2}$. Acta Math. 159 (1987), 215-259.

[7] Chen, W.; Li, C.: A priori estimates for prescribing scalar curvature equations. Ann. of Math. 145 (1997), 547-564.

[8] Chen, C. C.; Lin, C. S.: Prescribing scalar curvature on $\mathbb{S}^{N}$, I. A priori estimates. J. Differential Geom. 57 (2001), 67-171.

[9] Christoffel, E. B.: Ueber die Bestimmung der Gestalt einer krummen Oberfläche durch lokale Messungen auf derselben. J. für die reine und angew. Math., 64 (1865),193-209.

[10] Escobar, J. F.; Schoen, R. M.: Conformal metrics with prescribed scalar curvature. Invent. Math. 86 (1986), 243-254.

[11] Firey, W. J.: The determination of convex bodies from their mean radius of curvature functions. Mathematika 14 (1967), 1-13.

[12] Goodey, P., Yaskin, V., Yaskina, M., A Fourier transform approach to Christoffel's problem, Trans Amer Math Soc 363(2011), 6351-6384.

[13] Guan, P. F.; Ma, X. N.: The Christoffel-Minkowski problem I: Convexity of solutions of a Hessian equation. Invent. Math. 151 (2003), 553-577.

[14] Guan, P. F., Xia, C., $L_{p}$ Christoffel-Minkowski problem: the case $1<p<k+1$, Calc. Var. PDE. 57 (2018), no. 2, Art. 69, 23 pp. 
[15] Hilbert, D.: Grundzüge einer allgemeinen Theorie der linearen Integralgleichungen. Leipzig und Berlin, 1912.

[16] Hu, C.; Ma, X. N.; Shen, C.: On the Christoffel-Minkowski problem of Firey's p-sum, Calc. Var. PDE. 21 (2004), 137-155.

[17] Hurwitz, A.: Sur quelques applications géométriques des séries de Fourier. Ann. École Norm. 13 (1902), 357-408.

[18] Kazdan, J. L.; Warner, F.: Existence and conformal deformation of metrics with prescribed Gaussian and scalar curvatures. Ann. of Math. 101 (1975), 317-331.

[19] Pogorelov, A. V.: On the question of the existence of a convex surface with given sum of principal radii of curvature (in Russian). Uspehi Mat. Nauk 8 (1953), 127-130.

[20] Schneider, R.: Convex bodies: the Brunn-Minkowski theory. second expanded edition, Encyclopedia of Mathematics and its Applications, 151, Cambridge Univ. Press, 2014.

[21] Süss, W.: Bestimmung einer geschlossenen konvexen Fläche durch die Summe ihrer Hauptkrümmungsradien. Math. Ann. 108 (1933), 143-148.

Qi-Rui Li: School of Mathematical Sciences, Zhejiang University, Hangzhou 310027, China; and Centre for Mathematics and Its Applications, the Australian National University, Canberra, ACT 2601, Australia.

E-mail address: qi-rui.li@zju.edu.cn

Dongrui Wan: College of Mathematics and Statistics, Shenzhen University, Shenzhen 518060, CHINA

E-mail address: wandongrui@szu.edu.cn

Xu-Jia Wang: Centre for Mathematics and Its Applications, the Australian national University, Canberra, ACT 2601, Australia.

E-mail address: xu-jia.wang@anu.edu.au 